\title{
Complex Zeros of the Error Function and of the Complementary Error Function
}

\author{
By Henry E. Fettis, James C. Caslin and Kenneth R. Cramer
}

\begin{abstract}
The first one hundred zeros of the error function and of the complementary
\end{abstract} error function are given. An asymptotic formula for the higher zeros is also derived.

Introduction. The complementary error function is defined as

$$
\operatorname{Erfc}(z)=\frac{2}{\sqrt{ } \pi} \int_{z}^{\infty} e^{-t^{2}} d t \equiv 1-\operatorname{erf}(z)
$$

Closely associated with the above is the function $w(z)$ defined by

$$
w(z)=e^{-z^{2}} \operatorname{Erfc}(-i z),
$$

which has been studied and tabulated by numerous authors ([1], [2], [4], [5]). In certain physical problems relating to plasma instabilities, the function $i \pi^{1 / 2} w(z)$, often referred to as the "plasma dispersion function," is of importance. The function in this form has been tabulated by Fried and Conte [3]*, together with its first derivative, and, in this context, the zeros of the function play an important role. These zeros, which are symmetrically located with respect to the imaginary axis, lie entirely in the octants of the lower half-plane $2 \pi>\arg z>7 \pi / 4$ and $5 \pi / 4>\arg z>\pi$ and approach asymptotically the lines $y=-x$ and $y=x$, respectively.

Zeros of the ordinary error function have been computed previously by Salzer, but, to date, no extensive tabulation of the zeros of the complementary error function is known. Approximate values of the first two can be found from the altitude chart in $[1$, p. 298].

1. Properties of the Function $w(z)$. By definition, it is clear that

$$
\begin{aligned}
w(\bar{z}) & =\overline{w(-z)} ; \\
w(-z) & =2 e^{-z^{2}}-w(z) .
\end{aligned}
$$

From the above relations, it is possible to find $w(z)$ in the entire complex plane from its value in the first quadrant. In particular,

(a) $w(-x+i y)=\overline{w(x+i y)}$;

$$
\text { (b) } \quad w(x-i y)=2 e^{-(x-i y)^{2}}-\overline{w(x+i y)} \text {. }
$$

Received May 30, 1972.

AMS (MOS) subject classifications (1970). Primary 33A20.

* A more accurate table of this function has been prepared by the present authors and will be issued as an ARL Technical Report in the near future. See [9]. 
The following limiting forms are also evident:

$$
\begin{aligned}
& w(i y)=e^{y^{2}} \operatorname{Erfc}(y) ; \\
& w(x)=e^{-x^{2}}+\frac{2 i}{\pi^{1 / 2}} e^{-x^{2}} \int_{0}^{x} e^{t^{2}} d t .
\end{aligned}
$$

The last integral is sometimes called "Dawson's integral." For $x=y, w(x+i y)$ is expressible in terms of the Fresnel integrals

$$
\begin{aligned}
\text { (a) } C(x) & =\int_{0}^{x} \cos \frac{\pi}{2} t^{2} d t \\
\text { (b) } S(x) & =\int_{0}^{x} \sin \frac{\pi}{2} t^{2} d t \\
w[(1+i) x]= & e^{-2 i x^{2}}\left\{1-(1-i)\left[C\left(\frac{2 x}{\pi^{1 / 2}}\right)+i S\left(\frac{2 x}{\pi^{1 / 2}}\right)\right]\right\} .
\end{aligned}
$$

Some integral representations for $w(z)$, in addition to the one given by Eq. (1), are

$$
w(z)=\frac{i}{\pi} \int_{-\infty}^{\infty} \frac{e^{-t^{2}} d t}{z-t}, \quad \operatorname{Im}(z)>0,
$$

or, equivalently,

$$
w(z)=\frac{1}{\pi} \int_{-\infty}^{\infty} \frac{e^{-t^{2}}[y+i(x-t)] d t}{(x-t)^{2}+y^{2}}, \quad y>0 .
$$

The real part of $w(z)$ as defined by Eq. (9) is often referred to as the "Voigt function" and is of importance in astrophysics.

The function $w(z)$ may also be represented by the following continued fraction:

$$
w(z)=\frac{i}{\sqrt{ } \pi}\left[\frac{1}{z}-\frac{1 / 2}{z}-\frac{1}{z}-\frac{3 / 2}{z}-\frac{2}{z}-\cdots\right],
$$

as well as by the well-known power and asymptotic series for the error function. It is noted that neither the asymptotic series nor the continued fraction is valid if $z$ is in the lower half-plane. In this region, the function must be computed from Eq. (4)(b).

2. Location of the Zeros. Because of symmetry [Eq. (4)(a)], it is sufficient to consider $x>0$. By substituting $x-t=s$, Eq. (9) may be written in the equivalent form

$$
\begin{aligned}
w(z) & =u(x, y)+i v(x, y) \\
& =\frac{2 e^{-x^{2}}}{\pi}\left[y \int_{0}^{\infty} \frac{e^{-s^{2}} \cosh 2 x s}{y^{2}+s^{2}} d s+i \int_{0}^{\infty} \frac{e^{-s^{2}} \sinh 2 x s}{y^{2}+s^{2}} s d s\right]
\end{aligned}
$$

from which it is clear that $u(x, y)>0, v(x, y)>0$ for $x>0, y>0$. This, together with Eq. (4)(a), shows that any zeros of $w(z)$ which exist must lie in the lower half-plane and these, according to Eq. (4)(b), will be determined from the equations**

$$
2 e^{y^{2}-x^{2}} \cos 2 x y=u(x, y), \quad 2 e^{y^{2}-x^{2}} \sin 2 x y=-v(x, y)
$$

** Henceforth it will be assumed that $x$ and $y$ are positive. 
Since $u(x, y)$ and $v(x, y) \rightarrow 0$ for $|z| \rightarrow \infty$, it is evident that, asymptotically, $|y|<x$. Further, since $u$ and $v$ are both positive if $x$ and $y$ are positive, it follows that $\cos 2 x y>$ 0 , $\sin 2 x y<0$, and hence that

$$
2 x y=2 n \pi-\beta, \quad n=1,2, \cdots,
$$

where $0 \leqq \beta<\pi / 4$. Since, for large values of $|z|, w(z) \sim i / \pi^{1 / 2} z, \arg w \rightarrow \pi / 4$ as $\arg z \rightarrow \pi / 4$, and so $\beta \rightarrow \pi / 4$ as $n \rightarrow \infty$. Therefore, a reasonable assumption is $x \doteq \lambda+\alpha, y \doteq \lambda-\alpha$ where $\lambda=((n-1 / 8) \pi)^{1 / 2}$. From Eq. (12), we have

$$
2 e^{y^{2}-x^{2}}=|w| \text {. }
$$

In Eq. (14), the magnitude of $w$ can be approximated for large $|z|$ by the first term of the continued fraction:

$$
|w| \sim 1 /\left(\pi\left(x^{2}+y^{2}\right)\right)^{1 / 2}
$$

This gives

$$
2 e^{-4 \lambda \alpha} \doteq 1 / \sqrt{ } \pi\left[2 \lambda^{2}+2 \alpha^{2}\right]^{1 / 2} \doteq 1 /(2 \pi)^{1 / 2} \lambda
$$

Hence,

$$
\alpha \doteq \frac{1}{4 \lambda} \ln \left[2(2 \pi)^{1 / 2} \lambda\right]
$$

For a more precise estimate, we set

$$
x \doteq \lambda+\alpha+p, \quad y \doteq \lambda-\alpha+q .
$$

From Eq. (14), we then get, with the assumption that $\lambda p \ll 1, \lambda q \ll 1$,

$$
\begin{aligned}
2 e^{-4 \lambda \alpha}[1 & -2 p(\lambda+\alpha)+2 q(\lambda-\alpha)] \\
& \doteq \frac{1}{(2 \pi)^{1 / 2} \lambda\left[1+p(\lambda+\alpha) / \lambda^{2}+q(\lambda-\alpha) / \lambda^{2}\right]^{1 / 2}} \\
& \doteq \frac{1}{(2 \pi)^{1 / 2} \lambda}\left[1-\frac{p(\lambda+\alpha)}{2 \lambda^{2}}-\frac{q(\lambda-\alpha)}{2 \lambda^{2}}\right]
\end{aligned}
$$

whence, using (16),

$$
p\left[(\lambda+\alpha)\left(4 \lambda^{2}-1\right)\right]=q\left[(\lambda-\alpha)\left(4 \lambda^{2}+1\right)\right] .
$$

Thus,

$$
p \doteq q\left[1-2 \alpha / \lambda+1 / 2 \lambda^{2}\right]
$$

Also, from Eq. (12),

$$
\tan 2 x y=-v / u
$$

and, from Eq. (18),

$$
\begin{aligned}
2 x y & =2 \lambda^{2}-2 \alpha^{2}+2 p(\lambda-\alpha)+2 q(\lambda+\alpha)+2 p q \\
& \doteq 2 \lambda^{2}+2 \lambda(p+q)-2 \alpha^{2} .
\end{aligned}
$$


Using the addition formula for the tangent,

$$
\tan 2 x y \doteq-\frac{1-2 \lambda(p+q)+2 \alpha^{2}}{1+2 \lambda(p+q)-2 \alpha^{2}} .
$$

To approximate $\arg (w)$ adequately, we need two terms of the continued fraction:

$$
\pi^{1 / 2} w \doteq \frac{i z}{z^{2}-\frac{1}{2}}=\frac{(y-i x)}{\frac{1}{2}-(x+i y)^{2}}
$$

From (21),

$$
p \doteq q
$$

and, with this simplification, we get, from (24),

$$
-\tan 2 x y \doteq 1-8 \lambda p+4 \alpha^{2}
$$

and, from (25), after some manipulation

$$
\tan [\arg (w)] \doteq 1+2 \alpha / \lambda-1 / 2 \lambda^{2}
$$

Hence, equating the right sides, and solving

$$
p \doteq q \doteq \frac{2 \lambda \alpha^{2}-\alpha+(1 / 4 \lambda)}{4 \lambda^{2}}=\frac{8(\lambda \alpha)^{2}-4 \lambda \alpha+1}{16 \lambda^{3}}
$$

This gives the approximations

$$
\begin{aligned}
& x \doteq \lambda+\frac{1}{4 \lambda} \ln \left(2(2 \pi)^{1 / 2} \lambda\right)+\frac{1}{16 \lambda^{3}}\left\{1-\ln \left(2(2 \pi)^{1 / 2} \lambda\right)+\frac{1}{2}\left[\ln \left(2(2 \pi)^{1 / 2} \lambda\right)\right]^{2}\right\} \\
& y \doteq \lambda-\frac{1}{4 \lambda} \ln \left(2(2 \pi)^{1 / 2} \lambda\right)+\frac{1}{16 \lambda^{3}}\left\{1-\ln \left(2(2 \pi)^{1 / 2} \lambda\right)+\frac{1}{2}\left[\ln \left(2(2 \pi)^{1 / 2} \lambda\right)\right]^{2}\right\}
\end{aligned}
$$

where $\lambda=\left(\left(n-\frac{1}{8}\right) \pi\right)^{1 / 2}$.

Values obtained from the above approximation agree with the exact values to 3 figures for $n=0$ and to at least 8 figures for $n>50$.

It may be noted that the form of the three term asymptotic approximation to the roots of the more general equation $w(\bar{z})=a e^{-z^{2}}$ ( $a$ real and positive) is identical to Eq. (30) except that the logarithmic term is replaced by $\ln \left(a(2 \pi)^{1 / 2} \lambda\right)$. In particular, the choice $a=1$ gives the corresponding asymptotic approximation to the roots of $\operatorname{erf}(z)=0$, tabulated in [6].

3. Improvement of the Values Obtained by the Asymptotic Approximation. The approximate values given by the asymptotic approximation (30) may be used to obtain more accurate values by a method suggested by Salzer [6].

For convenience, we introduce the function $y(\rho)=\sqrt{ } \pi w(i \rho) / 2$. This function satisfies the differential equation

$$
d y / d \rho=2 \rho y-1
$$

or

$$
d \rho / d y=1 /(2 \rho y-1)
$$


Table $1-$ Zeros of $\operatorname{Erf}(z)$

\begin{tabular}{|c|c|}
\hline$N$ & $x$ \\
\hline $\begin{array}{l}1 \\
2 \\
3 \\
4 \\
5 \\
6 \\
7 \\
8 \\
9 \\
10\end{array}$ & 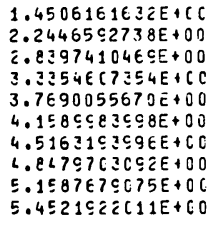 \\
\hline $\begin{array}{l}11 \\
12 \\
13 \\
14 \\
15 \\
16 \\
17 \\
18 \\
15 \\
20\end{array}$ & 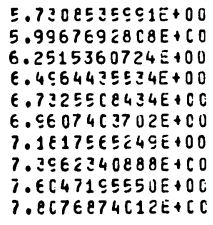 \\
\hline $\begin{array}{l}21 \\
22 \\
23 \\
24 \\
25 \\
26 \\
27 \\
28 \\
29 \\
30\end{array}$ & 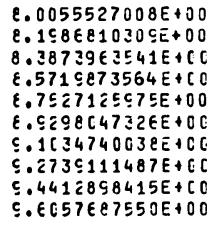 \\
\hline $\begin{array}{l}31 \\
32 \\
33 \\
34 \\
35 \\
3 E \\
37 \\
38 \\
39 \\
40\end{array}$ & 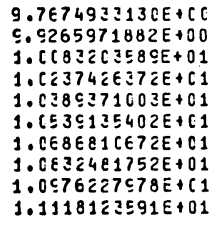 \\
\hline $\begin{array}{l}41 \\
42 \\
43 \\
44 \\
45 \\
46 \\
47 \\
48 \\
49 \\
50\end{array}$ & $\begin{array}{l}1.125823818 E E+01 \\
1.139 E E 37 C 98 E+C 1 \\
1.1533381775 E+C 1 \\
1.166853 C C 78 E+01 \\
1.180213 E E 7 E E+01 \\
1.1534252 E 02 E+C 1 \\
1.2 C 64 C 27481 E+C 1 \\
1.21942 C E 741 E+81 \\
1.2322134304 E+01 \\
1.244875165 E E+C 1\end{array}$ \\
\hline
\end{tabular}

\begin{tabular}{|c|c|c|}
\hline$Y$ & $N$ & $\cdot x$ \\
\hline $\begin{array}{l}1.8805436002 E+00 \\
2.6165751407 E+00 \\
3.1756280995 E+0 D \\
3 . E 4 E 17437 E 4 E+00 \\
4.0 E 06972339 E+00 \\
4.4355714442 E+00 \\
4.780447 E 442 E+00 \\
5.1 C 15880435 E+0 C \\
5.4 C 3332 E 420 E+00 \\
5.6888374370 E+0 C\end{array}$ & $\begin{array}{l}51 \\
52 \\
53 \\
54 \\
55 \\
56 \\
57 \\
58 \\
59 \\
E 0\end{array}$ & $\begin{array}{l}1.2574098205 E+01 \\
1.2658211423 E+01 \\
1.2321126973 E+01 \\
1.2942878851 E+01 \\
1.30 E 3499443 E+01 \\
1.3183019698 E+01 \\
1.3301469143 E+01 \\
1.3418876045 E+01 \\
1.3535267418 E+01 \\
1.36506 E 9147 E+01\end{array}$ \\
\hline 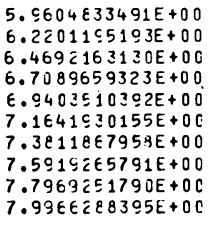 & $\begin{array}{l}51 \\
62 \\
\in 3 \\
64 \\
\in 5 \\
\in 6 \\
67 \\
\in 8 \\
\epsilon 9 \\
70\end{array}$ & $\begin{array}{l}1.37651 C 6033 E+01 \\
1.3878601857 E+01 \\
1.3951179441 E+01 \\
1.4102860 E 98 E+01 \\
1.4213666686 E+01 \\
1.4323617 E 49 E+01 \\
1.4432733065 E+01 \\
1.4541031 E 31 E+01 \\
1.4648531557 E+01 \\
1.4755250094 E+01\end{array}$ \\
\hline $\begin{array}{l}8.1914 \angle 87507 E+00 \\
8.3816701210 E+00 \\
8.5 E 7 E 5 C 4131 E+00 \\
8.749 E 7 C 17 E 4 E+0 D \\
8.527 \subseteq 477963 E+00 \\
9.1027134002 E+00 \\
\subseteq .2741 E 70843 E+0 C \\
9.4424 \subseteq C E 194 E+00 \\
9 . E 0784 C 7166 E+0 C \\
9.7703 \subseteq 59481 E+00\end{array}$ & $\begin{array}{l}71 \\
72 \\
73 \\
74 \\
75 \\
76 \\
77 \\
78 \\
79 \\
80\end{array}$ & $\begin{array}{l}1.48 E 1204074 E+01 \\
1.4966409685 E+01 \\
1.5070882551 E+01 \\
1.5174637762 E+01 \\
1.5277689897 E+01 \\
1.5380053048 E+01 \\
1.5481740841 E+01 \\
1.553276 E 4 E 1 E+01 \\
1.5683142669 E+01 \\
1.5782881821 E+01\end{array}$ \\
\hline $\begin{array}{l}9.5302 E 83819 E+00 \\
1.0087594981 E+01 \\
1.02424 C 380 J E+01 \\
1.0395 C 74059 E+01 \\
1.0545436985 E+01 \\
1 . C E C 3 E 7 E E E 4 E+01 \\
1.083 C E E 06 \subseteq 8 E+01 \\
1.0984130824 E+01 \\
1.112 E 5 C 3445 E+01 \\
1.1267070108 E+01\end{array}$ & $\begin{array}{l}81 \\
82 \\
83 \\
84 \\
85 \\
86 \\
87 \\
88 \\
89 \\
90\end{array}$ & $\begin{array}{l}1.5881995897 E+01 \\
1.59804964 E 6 E+01 \\
1.6078394805 E+01 \\
1.6175701808 E+01 \\
1.6272428058 E+01 \\
1.63 E 8583822 E+01 \\
1.64 E 4179072 E+01 \\
1.6559223491 E+01 \\
1.6653726485 E+01 \\
1.6747697200 E+01\end{array}$ \\
\hline $\begin{array}{l}1.1405897925 E+01 \\
1.1543 C 49948 E+01 \\
1.1678385507 E+01 \\
1.1812560508 E+01 \\
1.1545 C 27705 E+01 \\
1.2 C 76 C 36943 E+01 \\
1.2205 E 35379 E+01 \\
1.2333867680 E+01 \\
1.2460776202 E+01 \\
1.258 E 4 C 1155 E+01\end{array}$ & $\begin{array}{l}91 \\
62 \\
93 \\
c 4 \\
95 \\
95 \\
\subseteq 6 \\
67 \\
98 \\
99 \\
100\end{array}$ & $\begin{array}{l}1.6841144522 E+01 \\
1.6934077097 E+01 \\
1.7026503332 E+01 \\
1.7118431411 E+01 \\
1.7209869298 E+01 \\
1.73 C 0824747 E+01 \\
1.7351305311 E+01 \\
1.7481318347 E+01 \\
1.7570871025 E+01 \\
1.7659970332 E+01\end{array}$ \\
\hline
\end{tabular}

$1.2710780755 E+01$ $1.2833951354 E+01$ $1.2555947571 E+01$ $1.2555947571 E+01$
$1.30768024 C 1 E+C 1$ $1.30768024 C 1 E+C 1$
$1.3196547322 E+01$ $1.3196547322 E+01$
$1.3315212392 E+01$ $1.3432826332 E+01$ 1. $3549416616 E+01$ 1. $3665009537 E+01$ $1.3779630282 E+01$

$1.3893302996 E+01$ $1.400605084 \mathrm{CE}+01$ $1.400605084 C E+01$
$1.4117896045 E+01$ $1.4117896045 E+01$
$1.4228859967 E+01$ $1.4228859967 E+01$
$1.4338963132 E+01$ $1.4338963132 E+01$
$1.4448225279 E+01$ $1.455 E 665407 E+01$ $1.4664301807 E+01$ $1.4771152104 E+01$ $1.4877233285 E+01$

$1.4 \mathrm{C} 82561735 E+01$ $1.508715326 \mathrm{EE}+01$ $1.5191023142 E+01$ 1.52 $54186108 E+01$ $1.5396656414 E+01$ $1.5498447336 E+01$ 1. $5599573701 E+01$ $1.5700046903 E+01$ $1.579587992 E E+01$

$1.599767342 E E+01$ $1.6095656972 E+01$ $1.6193046511 E+01$ $1.6289852724 E+01$ $1.6386085974 E+01$ 1. $E 48175 E 323 E+01$ $1.657687353 C E+01$ $1.657 E 873535 E+01$ $1.671447114 E+01$ $1 . E 765486267 E+01$ $1.68589999 E 4 E+01$

1.655199692 CE+ 01 $1.7044485613 \mathrm{E}+01$ $1.713647429 C E+01$ 1.722797098 CE + 01 $1.731898349 E E+01$ 1.740051944CE+01 $1.7499586252 E+01$ $1.7495586252 E+01$ $1.758919112 E E+01$ $1.7678341112 E+01$
$1.7767043269 t+01$

Now, let $\rho_{n}$ be a zero of $y(\rho)$, and $\rho$ an approximation to $\rho_{n}$. Then, in the vicinity of $\rho$, we may expand $\rho_{n}$ into a power series in $y(\rho)$ :

$$
\rho=\rho_{n}+a_{1} y(\rho)+a_{2} y^{2}(\rho)+a_{3} y^{3}(\rho)+\cdots .
$$

Substituting (33) into (32), we get

$$
a_{1}=-1, \quad 2 a_{2}=2 a_{1} \rho_{n}, \quad 3 a_{3}=4 a_{2} \rho_{n}+2 a_{1}^{2}
$$

and, in general,

$$
k a_{k}=2\left[(k-1) \rho_{n} a_{k-1}+\sum_{i=1}^{k-2}(k-i-1) a_{i} a_{k-i-1}\right], \quad k>2 .
$$

The series up to terms of order $y^{4}$ is

$$
\rho_{n}=\rho+y(\rho)+\rho_{n} y^{2}(\rho)+\frac{4 \rho_{n}^{2}-2}{3} y^{3}(\rho)+\frac{4 \rho_{n}^{3}-5 \rho_{n}}{2} y^{4}(\rho)+\cdots .
$$

By successive substitutions, this may be expressed in terms of $\rho$ as follows: 
TABLE 2-Zeros of $\operatorname{Erfc}(z)$

\begin{tabular}{|c|c|}
\hline $\mathbf{N}$ & $x$ \\
\hline $\begin{array}{l}1 \\
2 \\
3 \\
4 \\
5 \\
6 \\
7 \\
8 \\
5 \\
10\end{array}$ & $\begin{array}{l}1.9 \subseteq 14668430 E+00 \\
E . E C 114 C C C 43 E+00 \\
3.235330 B E 84 E+C C \\
3.6 C 73097025 E+00 \\
4.1 C 61072847 E+00 \\
4.47 E 815 E C 3 C E+C C \\
4.8184882 C 19 E+0 C \\
5.137 C E 72713 E+0 C \\
E .4367 C 3 C 1 C 7 E+0 C \\
E .7204348510 E+00\end{array}$ \\
\hline $\begin{array}{l}11 \\
12 \\
13 \\
14 \\
15 \\
16 \\
17 \\
18 \\
19 \\
20\end{array}$ & 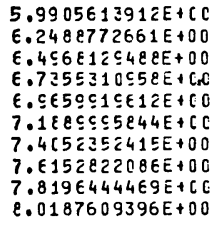 \\
\hline $\begin{array}{l}21 \\
22 \\
23 \\
24 \\
25 \\
26 \\
27 \\
28 \\
29 \\
30\end{array}$ & 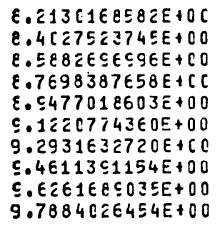 \\
\hline $\begin{array}{l}31 \\
32 \\
33 \\
34 \\
35 \\
36 \\
37 \\
38 \\
39 \\
40\end{array}$ & $\begin{array}{l}c .047 C 78025 C E+00 \\
1.0105021779 E+01 \\
1.025 \subseteq 650868 E+01 \\
1.0411 C 73515 E+01 \\
1.0562096 C 83 E+01 \\
1.0710093857 E+01 \\
1.085 E 071721 E+01 \\
1.160 C 1 C 475 \subseteq E+[1 \\
1.11422 E 8785 E+01 \\
1.128263480 E E+01\end{array}$ \\
\hline $\begin{array}{l}41 \\
42 \\
43 \\
44 \\
45 \\
46 \\
47 \\
48 \\
49 \\
50\end{array}$ & 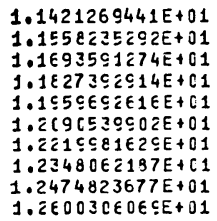 \\
\hline
\end{tabular}

\begin{tabular}{|c|c|c|}
\hline$Y$ & $N$ & $x$ \\
\hline $\begin{array}{l}-1.35431 C 1281 E+00 \\
-2.1770440061 E+00 \\
-2.784327 E 132 E+00 \\
-3.28741 C 7894 E+00 \\
-3.7259487194 E+00 \\
-4.116 E 3 E 227 E E+00 \\
-4.4798327977 E+00 \\
-4.81386 E B 20 E+00 \\
-5.1265315455 E+00 \\
-5.4215885769 E+00\end{array}$ & $\begin{array}{l}51 \\
52 \\
53 \\
54 \\
55 \\
56 \\
57 \\
58 \\
59 \\
60\end{array}$ & $\begin{array}{l}1.2724547357 E+01 \\
1.2847583687 E+01 \\
1.296944943+E+01 \\
1.305017756+E+01 \\
1.3269799235 E+01 \\
1.3328344397 E+01 \\
1.3445841623 E+01 \\
1.35 E 2316243 E+01 \\
1.3677800433 E+01 \\
1.3752313244 E+01\end{array}$ \\
\hline $\begin{array}{l}-5.701 E 5 E 445 E E+0 O \\
-5.9688 C C 2871 E+O C \\
-6.2246517451 E+0 D \\
-6.4705 Z 63755 E+00 \\
-6.7 C 75031267 E+0 C \\
-E .9364758 E 21 E+00 \\
-7.1582131926 E+00 \\
-7.3733477179 E+00 \\
-7.5824379224 E+00 \\
-7.7859648641 E+00\end{array}$ & $\begin{array}{l}61 \\
62 \\
63 \\
64 \\
E 5 \\
66 \\
67 \\
68 \\
89 \\
70\end{array}$ & $\begin{array}{l}1.3905930703 E+01 \\
1.4018525877 E+01 \\
1.4130270879 E+01 \\
1.4241136972 E+01 \\
1.4351144590 E+01 \\
1.44 E 0313386 E+01 \\
1.45 E 86 E 2274 E+01 \\
1.4676209468 E+01 \\
1.4782972518 E+01 \\
1.4888968340 E+01\end{array}$ \\
\hline $\begin{array}{l}-7.98434 \subseteq 0902 E+00 \\
-8.1779 E C 7741 E+00 \\
-8.3671277697 E+0 E \\
-8.5 E 21420331 E+00 \\
-8.7332651261 E+00 \\
-8.9107320199 E+00 \\
-9.0847551505 E+00 \\
-9.2555271305 E+00 \\
-9.4232232814 E+00 \\
-9.5880037302 E+00\end{array}$ & $\begin{array}{l}71 \\
72 \\
73 \\
74 \\
75 \\
16 \\
77 \\
78 \\
79 \\
80\end{array}$ & $\begin{array}{l}1.4994213254 E+01 \\
1.5098723005 E+01 \\
1.5202512799 E+01 \\
1.53 C 5597322 E+01 \\
1.54 C 7990768 E+01 \\
1.5509706862 E+01 \\
1.5610758880 E+01 \\
1.5711159 E E 8 E+01 \\
1.5810921665 E+01 \\
1.5910056918 E+01\end{array}$ \\
\hline $\begin{array}{l}-9.7500151899 E+00 \\
-9.9 C 93924807 E+00 \\
-1.0066259837 E+01 \\
-1.0220732033 E+01 \\
-1.0372 C 153 E 3 E+01 \\
-1.0522968493 E+01 \\
-1.067 C \varepsilon C 3202 E+01 \\
-1.081 E E \varepsilon 5041 E+01 \\
-1.0960633905 E+01 \\
-1.11067<4544 E+01\end{array}$ & $\begin{array}{l}81 \\
82 \\
83 \\
84 \\
85 \\
86 \\
87 \\
88 \\
89 \\
90\end{array}$ & $\begin{array}{l}1.6008577101 E+01 \\
1.6106493529 E+01 \\
1.6263817173 E+01 \\
1.6300558677 E+01 \\
1.6396728371 E+01 \\
1.6452336281 E+01 \\
1.6587392144 E+01 \\
1.6681905420 E+01 \\
1.6775885299 E+01 \\
1.68 E 9340713 E+01\end{array}$ \\
\hline $\begin{array}{l}-1.1243027016 E+01 \\
-1.1381607087 E+01 \\
-1.15185265 \subseteq 1 E+01 \\
-1.1653843753 E+01 \\
-1.1787 E 13474 E+01 \\
-1.191 \subseteq 88594 E+01 \\
-1.2650715120 E+01 \\
-1.218 C 14244 E E+01 \\
-1.2308213536 E+01 \\
-1.2434 \subseteq 70105 E+01\end{array}$ & $\begin{array}{r}91 \\
92 \\
93 \\
94 \\
65 \\
96 \\
67 \\
98 \\
99 \\
100\end{array}$ & $\begin{array}{l}1.69622803 E 4 E+01 \\
1.7054712689 E+01 \\
1.7146645915 E+01 \\
1.7238088045 E+01 \\
1.7329046863 E+01 \\
1.7419529576 E+01 \\
1.7569544756 E+01 \\
1.7599098410 E+01 \\
1.7688197956 E+01 \\
1.7776850238 E+01\end{array}$ \\
\hline
\end{tabular}

$-1.2560451772 E+01$ $-1.2 E 84 E 962 C 7 E+01$ $-1.2307739261 \mathrm{E}+01$ $-1.2 \$ 2 \subseteq 615080 E+01$ $-1.3050356257 E+01$ $-1.316999384 E E+C 1$ $-1.3288557544 E+01$ $-1.3280557544 E+01$ $-1.340 E 075732 E+01$ -1.352257556EE+ 01 $-1.3 E 38083042 E+01$

$-1.3752623072 E+01$ $-1.3866219544 E+01$ $-1.3978895378 E+01$ $-1.4090672581 E+01$ $-1.420157230 \mathrm{CE}+01$ $-1.4311614862 E+01$ $-1.43116140 E 2 E+01$ $-1.4420819825 E+01$ $-1.4529206013 E+01$ $-1.4636791555 E+01$ $-1.4743593923 E+01$

$-1.484 \subseteq E 29961 E+01$ $-1.495491592 \mathrm{CE}+01$ $-1.5059467483 E+01$ $-1.516329979 E E+01$ - $1.516329979 E E+01$ - $1.526 \angle 42748$ CE +01 $-1.5368864707 E+01$ $-1.5470525123 E+01$ $-1.5571721970 E+01$ $-1.5 E 72168051 E+01$ $-1.5771975767 E+01$

$-1.587115712 E E+01$ $-1.5969723768 E+01$ $-1.6067686975 E+01$ - $1.60676057680 \mathrm{O}$ - $1 . E 1 E 505768 \mathrm{EE}+01$ $-1.626184652 \angle E+01$ $-1.6358063780 E+\Pi 1$ $-1.6453719462 E+01$ $-1.6548823284 E+C 1$ $-1 . E 64338468 \mathrm{CE}+01$ $-1.6737412822 E+91$

$-1.6830916625 E+01$ $-1 . E 9239047 E C E+01$ - 1.E -1.7 $-1.7108367530 E+01$ - $1.719585835 E E+01$ $-1.7250865924 E+01$ $-1.7381397797 \bar{E}+01$ $-1.7471461356 E+01$ $-1.7561063792 E+01$ $-1.7 E 5 C 212111 E+01$

$$
\rho_{n}=\rho+y+\rho y^{2}+\frac{4 \rho^{2}+1}{3} y^{3}+\frac{12 \rho^{3}+7 \rho}{6} y^{4}+\cdots
$$

It is interesting to note that the iteration scheme

$$
\rho_{n}^{(k+1)}=\rho_{n}^{(k)}+y\left(\rho_{n}^{(k)}\right)+\rho_{n}^{(k)}\left[y\left(\rho_{n}^{(k)}\right)\right]^{2}
$$

based on Eq. (37) differs in the third term from that obtained by Newton's method, namely

$$
\rho_{n}^{(k+1)}=\rho_{n}^{(k)}+y\left(\rho_{n}^{(k)}\right)\left\{1+2 \rho_{n}^{(k)} y\left(\rho_{n}^{(k)}\right)+\cdots\right\} .
$$

The accompanying table lists the first hundred zeros of $w(z)$ and of $\operatorname{erf}(z)$. In computing these, values of $w(z)$ were obtained by numerical integration of Eq. (9). The error estimate for this type of quadrature is given by the Poisson summation formula. The resulting formulae are essentially the same as Salzer's (see [7], [8]).

For $n \geqq 5$, only the first three terms of (37) are required, and for $n \geqq 0$ at most four terms are needed, except $n=0$, where the fifth term contributes 2 units in the last figure of the real part. Comparison of the first ten of the values obtained for the 
zeros of $\operatorname{erf}(z)$ with those given by Salzer [6] disclosed only two discrepancies, one amounting to one unit in the tenth figure of the real part of the second zero and the other to one unit in the tenth figure of the imaginary part of the eighth one.

Acknowledgment. The authors are indebted to Professor Walter Gautschi, Department of Computer Sciences, Purdue University, and to Dr. Herbert E. Salzer for reading the preliminary manuscript and offering numerous constructive criticisms.

Aerospace Research Laboratories Wright-Patterson Air Force Base Ohio 45433

1. M. Abramowitz \& I. A. Stegun (Editors), Handbook of Mathematical Functions, With Formulas, Graphs and Mathematical Tables, Nat. Bur. Standards Appl. Math. Series, 55, Superintendent of Documents, U.S. Government Printing Office, Washington, D.C., 1964, Chap. 7. MR 29 \#4914.

2. V. N. Faddeeva \& N. M. Terent'Ev, Tables of Values of the Function $w(z)=e^{-z^{2}} \times$ $\left(1+2 i \pi^{-1 / 2} \int_{0}^{z} e^{t 2} d t\right)$ for Complex Argument, GITTL, Moscow, 1954; English transl., Math. Tables Series, vol. 2, Pergamon Press, Oxford, 1961. MR 16, 960; MR 22 \# 12740.

3. B. D. Fried \& S. D. Conte, The Plasma Dispersion Function. The Hilbert Transform of the Gaussian, Academic Press, New York, 1961. MR 24 \#B1958.

4. K. A. KARPov, Tables of the Function $w(z)=e^{-z^{2}} \int_{0}^{z} e^{x^{2}} d x$ in a Complex Region, Izdat. Akad. Nauk SSSR, Moscow, 19:54; Akad. Nauk SSSR. Vyčisl. Centr Mat. Tablicy, Izdat. Akad. Nauk SSSR, Moscow, 1958. (Russian) MR 16, 749; MR 24 \#B1297.

5. C. William MaRTZ, Tables of the Complex Fresnel Integral, NASA Report SP-3010, Washington, 1964.

6. H. E. SAlzer, "Complex zeros of the error function," J. Franklin Inst., v. 260, 1955, pp. 209-211. MR 17, 197.

7. H. E. SALZER, "Formulas for calculating the error function of a complex variable," $M T A C$, v. 5, 1951, pp. 67-70. MR 13, 989; 1140 .

8. H. E. FETTIS, "Numerical calculation of certain definite integrals by Poisson's summation formula," $M T A C$, v. 9, 1955, p. 88. MR 17, 302 .

9. Henry E. Fettis, James C. Caslin \& Kenneth R. Cramer, An Improved Tabulation of the Plasma Dispersion Function. Parts I and II, ARL 72-0056, 72-0057, May 1972.

10. Kenneth R. CRamer, Elmax Low Frequency Instabilities, ARL 72-0078, May 1972. 